\title{
Familial Disorders of the Optic Disc: Presentation of a Mother and Daughter and Review of the Literature
}

\author{
Chia Lee Hsu ${ }^{a} \quad$ Christopher J. Layton ${ }^{\mathrm{a}-\mathrm{c}}$ \\ ${ }^{a}$ Ophthalmology Unit and ${ }^{b}$ Gallipoli Medical Research Institute, Greenslopes Private \\ Hospital, and 'The University of Queensland School of Medicine, Brisbane, Qld., Australia
}

\section{Key Words}

Coloboma $\cdot$ Optic disc pit $\cdot$ Macular retinoschisis

\begin{abstract}
Optic disc pit and optic nerve coloboma are examples of congenital optic disc abnormalities. Although optic nerve coloboma can be inherited in an autosomal dominant fashion, no conclusive link has been found in the case of optic disc pit as an autosomal dominant disease. We describe two cases: a daughter with an optic disc pit complicated by maculopathy and her mother with a congenitally abnormal optic disc complicated by peripapillary choroidal neovascularisation.

(c) 2015 S. Karger AG, Basel
\end{abstract}

\section{Case Presentations}

Case 1

An asymptomatic 30-year-old female with no ocular history was referred by her optometrist with an abnormal macula optical coherence tomography (OCT) result. In her history, she reported having a brother who is blind from encephalitis. She also reported a history of trauma while on duty as a police officer, and she had been recently treated for a period of 8 weeks with antiepileptic treatment.

On ocular examination, the patient had Snellen visual acuities of $6 / 6$ in the right eye and $6 / 5$ in the left eye, with a near unaided distance of $\mathrm{N} 4.5$. She had good optic nerve functions with normal pupillary light responses, no relative afferent pupillary defect and full colour

KARGER125:s $12 \begin{aligned} & \text { Chia Lee Hsu, BMedSc (Hons), MBBS } \\ & \text { Ophthalmology Unit } \\ & \text { Greenslopes Private Hospital } \\ & \text { Brisbane, QLD 4120 (Australia) } \\ & \text { E-Mail hsuchialee@gmail.com }\end{aligned}$


Hsu and Layton: Familial Disorders of the Optic Disc: Presentation of a Mother and Daughter and Review of the Literature

vision on Ishihara plate testing. Goldmann applanation tonometry showed intraocular pressures of $18 \mathrm{~mm} \mathrm{Hg}$ in her right eye and $16 \mathrm{~mm} \mathrm{Hg}$ in her left eye. She had full ocular motility. Slit-lamp examination of the anterior segment and adnexa was also unremarkable.

Fundoscopic examination showed bilaterally large optic discs measuring $2 \mathrm{~mm}$ in diameter, a temporal optic disc pit (ODP) and loss of the foveal depression in the right eye, and retinal pigment epithelium changes at the macula. Subsequent diagnostic OCT (fig. 1) confirmed right-sided macular intraretinal fluid associated with the temporal disc pit. Fundus fluorescein angiogram (FFA; fig. 1) showed diffuse fluorescein leakage from the optic disc towards the macula.

The patient's right-sided vision deteriorated to 6/9 on her 1-week follow-up appointment, and submacular fluid increased. Due to the location of the intraretinal oedema, subthreshold argon laser photocoagulation in the peripapillary macular bundle adjacent to the disc was performed.

On her 3-month follow-up visit, fundoscopic examination showed re-formation of the foveal pit and no progression in the pre-existing pigmentary changes at the right foveal pit. OCT scanning confirmed resolution of her subretinal fluid. Visual acuity remained 6/9 in her right eye and 6/5 in her left eye. The 1-year follow-up showed no re-accumulation of fluid on OCT scan (fig. 1) and stable visual acuity.

\section{Case 2}

Her 61-year-old type 2 diabetic mother was referred 6 months later as a glaucoma suspect due to her left disc appearance and perimetry, which revealed a left superior arcuate defect.

On ocular examination, her Snellen visual acuities were 6/6 in the right eye and 6/5 in the left eye. Optic nerve functions were normal, evident by good pupillary light responses, no relative afferent pupillary defect and full colour vision on Isihara plate testing. Slit-lamp examination revealed non-visually significant nuclear sclerosis and open anterior chamber angles. Goldmann applanation tonometry revealed normal intraocular pressures of $16 \mathrm{~mm}$ $\mathrm{Hg}$ bilaterally. Dilated fundus examination showed a normal right optic disc and fundus and a larger, congenitally abnormal left optic disc (fig. 2) with associated inferior thinning of the retinal nerve fibre layer and peripapillary subretinal blood suggestive of a peripapillary choroidal neovascular membrane.

OCT scanning did not reveal associated retinal oedema, and subsequent FFA (fig. 3) revealed inferior disc hyperfluorescence and an inferior peripapillary choroidal neovascular membrane not threatening the macula. Unfortunately, both patients declined nextgeneration gene sequencing or specific testing for genes associated with ocular development such as PAX2 or PAX6. No other family members were available for examination.

Due to the location of the peripapillary choroidal neovascularisation and its low level of associated retinal oedema, the patient was offered ongoing active observation. Serial OCT, perimetry and FFA performed after 6 months showed no change or progression of her condition, with visual acuities remaining at $6 / 6$ in the right eye and $6 / 5$ in the left.

\section{Congenital Optic Disc Anomalies Associated with Normal or Large Discs}

Congenital optic nerve anomalies associated with normal or large optic disc include megalopapilla, morning glory disc, optic disc coloboma, ODP and tilted disc. Visual prognosis can range from asymptomatic to total blindness [1]. Presentation can occur as an isolated finding, in association with other ocular abnormalities or as part of a systemic disorder. Con- 
Hsu and Layton: Familial Disorders of the Optic Disc: Presentation of a Mother and Daughter and Review of the Literature

genital optic nerve anomalies are distinguished on fundus examination by an abnormal disc size, abnormalities in disc conformation, and the presence of abnormal tissue within or around the disc. Central nervous system malformations are common in patients with abnormal optic discs [2], and magnetic resonance imaging, a non-invasive neuroimaging modality, is sometimes necessary to identify associated central nervous system disorders and to predict the prognosis of neurodevelopment and endocrinological problems [3].

Megalopapilla is characterized by an abnormally large optic disc with a high cup-to-disc ratio. This condition is considered a normal physiological variant as it has a normal retinal nerve fibre layer. However, poor visual acuity has been reported in association with megalopapilla [4].

The morning glory disc abnormality is another congenital condition in which an enlarged, pale, concave disc is surrounded by chorioretinal pigmentary changes and radial spoke vessels said to resemble a morning glory flower [5]. The centre portion of the disc is occupied by a white clump of glial tissue. This defect is usually unilateral, occurs more commonly in females and causes a variable degree of visual deficiency, usually due to its associated foveal aplasia [2]. Visual impairment can also occur due to complications such as serous retinal detachment, retinal folds and subretinal neovascularisation [5].

Optic nerve coloboma is caused by incomplete closure of the embryonic fissure and presents as an isolated finding or in association with developmental syndromes such as Aicardi syndrome, CHARGE syndrome, renal coloboma syndrome and trisomy 13 . The condition can arise sporadically or by autosomal dominant inheritance. The defect can be observed fundoscopically as a sharp white decentred bowl-shaped excavation of the optic disc [6]. The optic disc is typically enlarged with a thin or absent inferior neuroretinal rim, rarely affecting the entire disc. It can be associated with coloboma of the retina, ciliary body or iris [2]. Visual prognosis depends mainly on the involvement of the papillomacular bundle and the severity of complications, which include choroidal neovascularisation and retinal detachment when there is an associated retinal coloboma. Histological study has revealed the presence of intrascleral smooth muscle substance around the distal optic nerve [3]. In our opinion, this is the most likely underlying cause of the congenital disc abnormality observed in case 2 .

ODP is another disc abnormality and can be seen as a grayish oval or round depression, usually detected in the inferotemporal part of the optic disc, with $20 \%$ affecting the central disc and $10 \%$ located in the other parts of the disc. The long-standing subretinal fluid affecting the temporal edge causes adjacent changes in the retinal pigment epithelium which often alerts the examiner to its presence. ODPs can arise as a congenital abnormality, with an incidence of 1 in 11,000 people, and occurs equally in women and men. Some patients with congenital optic nerve pit remain asymptomatic throughout life, but $25-75 \%$ present with ODP maculopathy in their 30-40s, a common complication associated with visual deterioration [7]. While the majority of the cases present unilaterally, 15\% of congenital ODPs are bilateral. More than one pit can occasionally occur. ODPs may be associated with optic nerve head coloboma and large optic discs. It differs from optic disc coloboma because it does not affect the disc margin. Acquired ODPs (AODPs) are usually associated with glaucoma. Morphologically, it is difficult to distinguish the congenital subtype; however, it more commonly affects females than males and is detected more often at the inferior disc. This condition can affect both eyes in up to $48 \%$ of patients and can be associated with scotomas. Interestingly, the prevalence of AODP is higher in patients with low-tension glaucoma compared to those with high-tension glaucoma. Disc haemorrhage was also more common in glaucoma patients with AODP (40\%) than in those without (8\%). Maculopathy is not a complication of AODP, although there have been suggestions that untreated glaucoma may result in worsening of the depth and width of the cup, allowing choroidal leakage into the subretinal space [7]. 
Hsu and Layton: Familial Disorders of the Optic Disc: Presentation of a Mother and Daughter and Review of the Literature

Histologic studies on congenital ODPs have revealed extension of dysplastic retina through a defect in the lamina cribosa into the subarachnoid space, with strands of condensed vitreous at the margin of the pit. Nerve axons are seen crossing over the pit [8]. Congenital ODPs have been associated with several systemic conditions such as Aicardi syndrome and Alagille syndrome. ODPs have also been linked to bilateral renal hypoplasia and some neurological developmental malformations [7]. Previous studies have suggested that unilateral ODPs may be inherited in an autosomal dominant pattern, although most tend to appear sporadically [9]. Stefko et al. [9] studied the relation of PAX2 gene mutation for a family of three generations with unilateral optic pits and concluded that no mutations of that gene were found, but a presumptive autosomal dominant pattern was still observed.

Macular retinoschisis and serous macular detachment have been described as complications of congenital optic nerve abnormalities, with controversies surrounding two issues: the source of the fluid and the mechanism of retinoschisis [10]. Several possible sources of this fluid have been postulated; fluid originating from the subarachnoid space, fluid from liquefied vitreous, fluid from leaky blood vessels at the base of the pit and fluid from the orbital space and surrounding dura [7]. However, the source remains controversial, and these studies have failed to demonstrate conclusive evidence. Several proposed mechanisms leading to the maculopathy have also been suggested. Firstly, the presence of small defects overlying the pit was thought to serve as a passage of fluid into the subretinal space. Secondly, liquefied vitreous overlying the optic nerve might cause tractional forces that can induce retinal shearing, allowing fluid to dissect through the layers [7]. Some patients have membrane spanning over the abnormal ODPs shown on OCT scans [11], and this membrane has been proposed to be a protective factor against the development of maculopathy [8]. Regardless of the origin of the fluid and the mechanism of retinoschisis, a study using highresolution OCT showed that fluid can move directly from the optic pit into the layers of the retina. The outer nuclear layer was found to be the most commonly affected layer [12].

The diagnosis of OCP is made mainly on fundus examination and OCT imaging. The inferotemporal depression with or without an overlying membrane can be seen on fundus examination. Macular elevation or a hole in the other retinal layer can also be seen. OCT scans can show the detachment with a schisis cavity or retinal oedema within the layers. Vitreomacular traction, vitreous strands and a diaphanous membrane overlying the disc can also be detected with OCT [12].

The treatment for ODP maculopathy remains controversial. Studies have shown that conservative management of ODP-related maculopathy is associated with a poor outcome [13]. As such, surgical approaches have become the preferred treatment modality for many practitioners. Argon laser photocoagulation between the detached retina and the optic nerve has been used to seal and minimize the communication from the pit to the subretinal space. In our case, this method has proven to be useful in achieving resolution and sustained macular reattachment and, subsequently, maintenance of the patient's visual acuity. Vitrectomy, with or without internal limiting membrane peel, and gas tamponade have also been shown to improve vision in several cases [7]. One recent report involving a 44-year-old woman with persistent ODP maculopathy showed positive results from an adjunctive autologous platelet treatment [14].

In our first case, an ODP was observed in association with a chronic maculopathy. OCT confirmed an ODP-associated maculopathy with sub- and intraretinal fluid, and FFA showed hyperfluorescence extending from the pit margin to the area of the schisis cavity. The patient did not have any other signs or symptoms of systemic disease that could account for macular retinoschisis or central serous retinopathy, such as Waldenstrom macroglobulinaemia, mul- 
Hsu and Layton: Familial Disorders of the Optic Disc: Presentation of a Mother and Daughter and Review of the Literature

tiple myeloma, leukaemia, age-related macular degeneration, Vogt-Koyanagi-Harada disease, hypertension or steroid use [11].

Several possible explanations for the findings of two disc anomalies in one family are hypothesised. This small familial case series could indeed represent coincidental findings of independent disc anomalies in two different individuals, without any true association. However, the presence of these unusual findings in a mother and daughter certainly suggests a possible genetic link between the two structural anomalies, but such a conclusion would need further genetic studies for confirmation.

\section{Summary}

These cases are interesting because they present a mother and a daughter each with different congenital unilateral optic disc anomalies. Case 1 showed a grayish temporal ODP associated with retinoschisis and macular detachment, which responded well to subthreshold peripapillary laser. Case 2 presented a clearly congenitally anomalous optic disc associated with peripapillary neovascularisation, which may have been caused by an optic nerve coloboma. The close genetic relationship between the patients presented may support the proposal that the two different congenital optic disc anomalies have a common inherited component within this family. Unfortunately, as this is only a small case series, we would not recommend genetic testing among family members.

\section{Statement of Ethics}

Both mother and daughter described above have provided informed consent for the publication of this report.

\section{Disclosure Statement}

The authors declare no conflict in interest.

\section{References}

1 Daroff R, Bradley W: Bradley's Neurology in Clinical Practice. Philadelphia, lsevier/Saunders, 2012.

2 Golnik K: Congenital optic nerve anomalies. Curr Opin Ophthalmol 1998;9:18-26.

-3 Brodsky M: Congenital optic disk anomalies. Surv Ophthalmol 1994;39:89-112.

4 Beck RW, Smith CH: Neuro-Ophthalmology: A Problem-Oriented Approach. Boston, Little Brown, 1998, pp 183-195.

5 Sobol W, Bratton A, Rivers M, Weingeist T: Morning glory disk syndrome associated with subretinal neovascular membrane formation. Am J Ophthalmol 1990;110:93-94.

6 Brodsky MC: Congenital anomalies of the optic disc; in Miller NR, Newman NJ (eds): Walsh \& Hoyt's Clinical Neuro-Ophthalmology, ed 5. Baltimore, Williams and Wilkins, 1998, pp 775-823.

7 Georgalas I, Ladas I, Georgopoulos G, Petrou P: Optic disc pit: a review. Graefes Arch Clin Exp Ophthalmol 2011;249:1113-1122.

-8 Ferry A: Macular detachment associated with congenital pit of the optic nerve head. Arch Ophthalmol 1963;70:346.

-9 Stefko S, Campochiaro P, Wang P, Li Y, Zhu D, Traboulsi E: Dominant inheritance of optic pits. Am J Ophthalmol 1997;124:112-113.

10 Sobol W, Blodi C, Folk J, Weingeist T: Long-term visual outcome in patients with optic nerve pit and serous retinal detachment of the macula. Ophthalmology 1990;97:1539-1542. 
Hsu and Layton: Familial Disorders of the Optic Disc: Presentation of a Mother and Daughter and Review of the Literature

11 Moreno-López M, González-López JJ Jarrín E, Bertrand J: Retinoschisis and macular detachment associated with acquired enlarged optic disc cup. Clin Ophthalmol 2012;6:433.

12 Imamura Y, Zweifel S, Fujiwara T, Freund K, Spaide R: High-resolution optical coherence tomography findings in optic pit maculopathy. Retina 2010;30:1104-1112.

13 Sugar H: Congenital pits in the optic disc. Am J Ophthalmol 1967;63:298-307.

14 Rosenthal G, Bartz-Schmidt K, Walter P, Heimann K: Autologous platelet treatment for optic disc pit associated with persistent macular detachment. Graefes Arch Clin Exp Ophthalmol 1998;236:151-153.

15 Ragge N, Ravine D, Wilkie A: Dominant inheritance of optic pits. Am J Ophthalmol 1998;125:124. 


\section{Case Reports in Ophthalmology}

\begin{tabular}{l|l}
\hline \multicolumn{2}{l}{ Case Rep Ophthalmol 2015;6:251-259 } \\
\hline DOI: 10.1159/000437381 & $\begin{array}{l}\text { ○ 2015 S. Karger AG, Basel } \\
\text { www.karger.com/cop }\end{array}$ \\
\hline
\end{tabular}

Hsu and Layton: Familial Disorders of the Optic Disc: Presentation of a Mother and Daughter and Review of the Literature
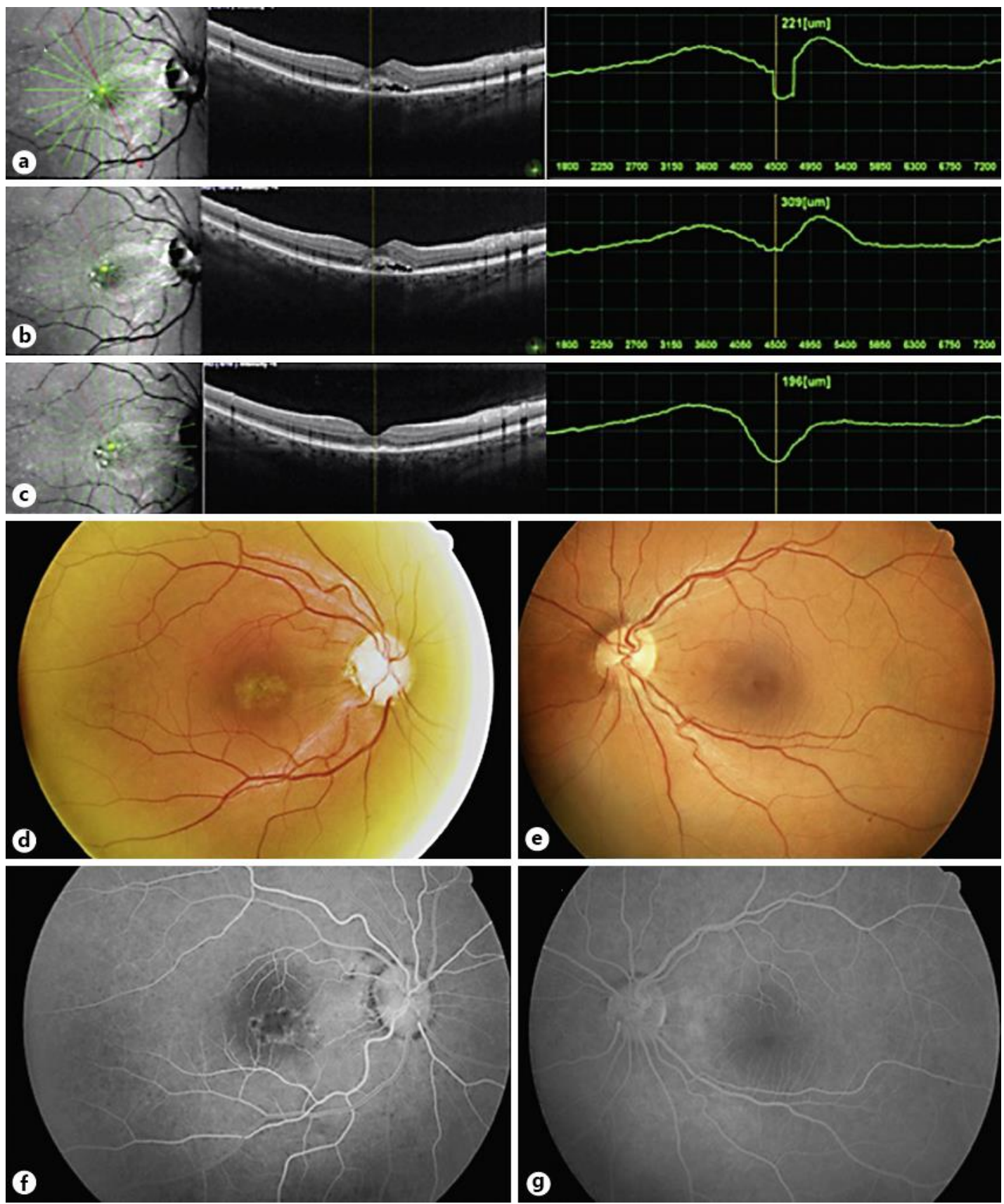

Fig. 1. Case 1. a Right eye radial OCT revealing macular retinoschisis and detachment associated with optic nerve pit on the first visit. b One-week serial radial OCT showing an increase in macular thickness with ongoing maculopathy. c One-year post-treatment radial OCT showing resolution of maculopathy and macular attachment. d, e Fundus photographs of the right and left eye at presentation, showing maculopathy in the right eye with associated temporal ODP. The pit is seen as a hyperpigmented depression. $\mathbf{f}, \mathbf{g}$ The right eye showed diffuse fluorescein leakage migrating from the optic disc defect towards the macula. 


\section{Case Reports in \\ Ophthalmology}

\begin{tabular}{l|l}
\hline \multicolumn{2}{l}{ Case Rep Ophthalmol 2015;6:251-259 } \\
\hline DOI: $10.1159 / 000437381$ & $\begin{array}{l}\text { ○ 2015 S. Karger AG, Basel } \\
\text { www.karger.com/cop }\end{array}$ \\
\hline
\end{tabular}

Hsu and Layton: Familial Disorders of the Optic Disc: Presentation of a Mother and Daughter and Review of the Literature

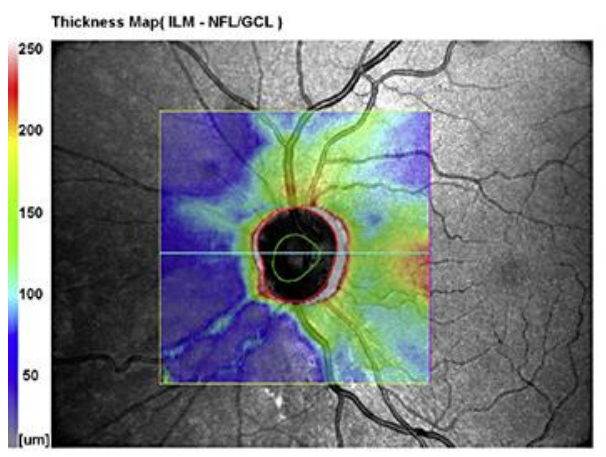

OCT( Horizontal 67 / 128)

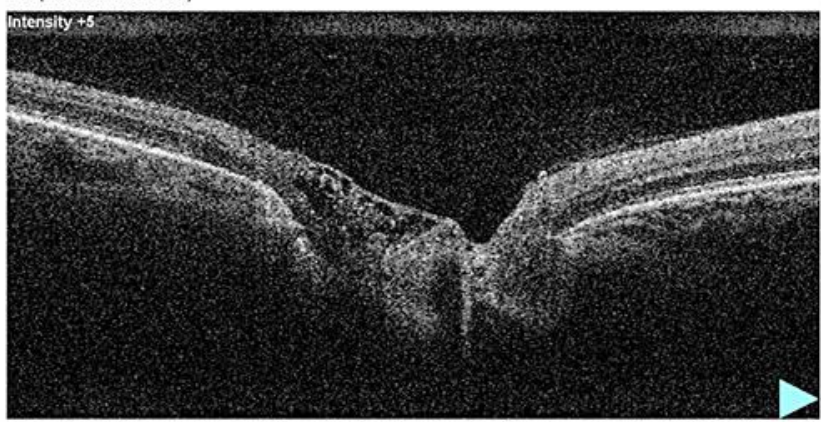

Fig. 2. a Radial OCT picture of the abnormal ODP in case 1. b Radial OCT picture of the abnormal left optic disc in case 2. 


\section{Case Reports in \\ Ophthalmology}

\begin{tabular}{l|l}
\hline Case Rep Ophthalmol 2015;6:251-259 \\
\hline DOI: $10.1159 / 000437381$ & $\begin{array}{l}\text { C 2015 S. Karger AG, Basel } \\
\text { www.karger.com/cop }\end{array}$ \\
\hline
\end{tabular}

Hsu and Layton: Familial Disorders of the Optic Disc: Presentation of a Mother and Daughter and Review of the Literature
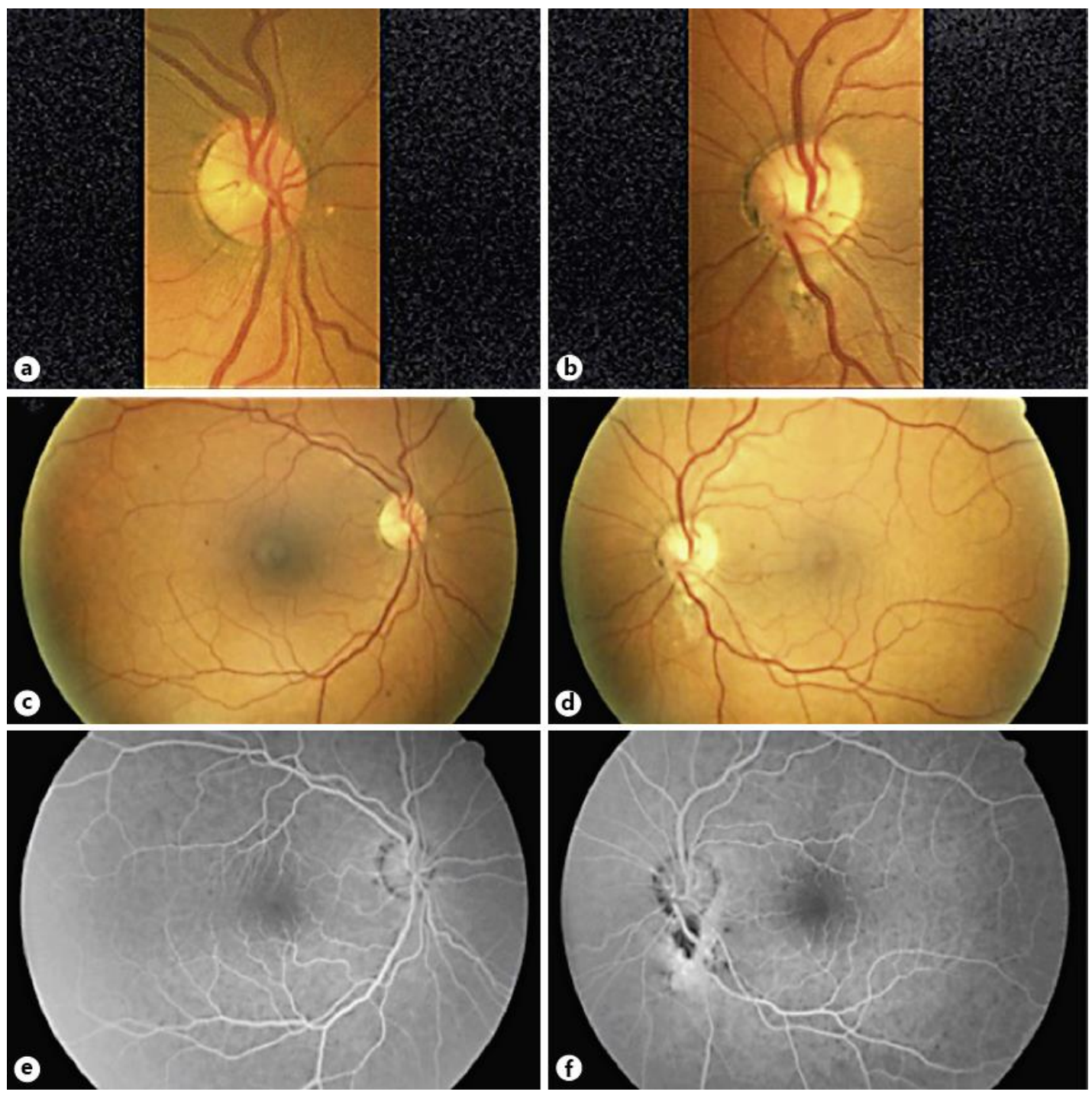

Fig. 3. a Normal right optic disc. b Congenitally abnormal left optic disc with an inferior hypopigmented area surrounded by an area of hyperpigmentation and subretinal blood. c Normal right fundus photograph. $\mathbf{d}$ Left fundus photograph showing the abnormal optic disc. Note that this optic disc is larger in size compared to the right optic disc. e Normal right FFA. f Peripapillary hypofluoresence with surrounding fluorescein leakage increasing in size and extent throughout the study. 